Concerns from Community

Mental Health Center (CMHC)

Patients Regarding Drug-Induced Movement Disorders: Impact on Functioning and Treatment Beliefs

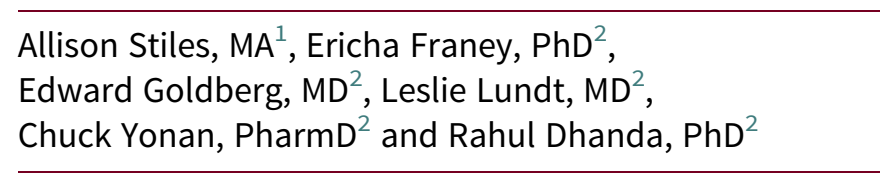

${ }^{1}$ CommonGround Program, Byfield, MA, USA, and ${ }^{2}$ Neurocrine Biosciences, Inc., San Diego, CA, USA

Presenting Author: Rahul Dhanda

\begin{abstract}
Objective. Drug-induced movement disorders (DIMDs) may occur in patients treated with antipsychotics. The CommonGround Program supports the recovery and healing of psychiatric outpatients through tools which facilitate better patient-doctor communication regarding psychiatric symptoms, DIMDs, and the effectiveness of treatment.

Methods. Patients responses to CommonGround's web-based waiting-room questionnaire were analyzed in patients who responded yes to having concerns about developing DIMDs (MD-YES) and those who responded no to this question (MDNO). These groups were compared descriptively to assess the potential effects of DIMD concerns on self-reported functioning and beliefs about prescribed psychiatric medications.

Results. Of 7874 responding patients, $312(4.0 \%)$ and 7562 (96.0\%) were in the MD-YES and MD-NO subgroups, respectively. A higher percentage of MD-YES patients reported poor / not so good ability to keep up with daily responsibilities ( $21.2 \%$ vs $15.2 \%$ vs MD-NO), along with low energy levels ( $37.1 \%$ vs $26.3 \%$ for MD-NO), bothersome thoughts/beliefs/fears (30.5\% vs $16.0 \%$ ), and nervousness/anxiety (35.3\% vs $27.5 \%$ ) all / most of the time. MD-YES patients were also more likely to wonder about stopping their medications ( $9.3 \%$ vs $0.6 \%$ for $\mathrm{MD}-\mathrm{NO}$ ) and were concerned about side effects such as sleepiness (31.4\% vs $3.9 \%)$ and weight gain (37.2\% vs $5.7 \%)$.

Conclusion. Patients from community mental health centers who were concerned about developing DIMDs tended to express problems with daily functioning and concerns about their psychiatric medications. For these patients, recognizing their fears and concerns may help clinicians discuss treatment options for DIMDs, which could increase patient confidence, encourage adherence to current psychiatric medications, and potentially improve outcomes.
\end{abstract}

\title{
Using Item 8 of the Abnormal Involuntary Movement Scale (AIMS) to Assess Improvement in Patients with Tardive Dyskinesia
}

\section{Leslie Citrome, $\mathrm{MD}^{1}$, Leslie Lundt, $\mathrm{MD}^{2}$, Chirag Shah, PharmD ${ }^{2}$ and Tara Carmack, MS $^{2}$} ${ }^{1}$ New York Medical College, Valhalla, NY, USA, and ${ }^{2}$ Neurocrine Biosciences, Inc.,
San Diego, CA, USA

Presenting Author: Leslie Citrome 